\title{
Nonlinear Optical Properties of Core-Shell Nanocavities for Enhanced Second-Harmonic Generation
}

\author{
Ye Pu, ${ }^{1, *}$ Rachel Grange, ${ }^{1}$ Chia-Lung Hsieh, ${ }^{1,2}$ and Demetri Psaltis ${ }^{1}$ \\ ${ }^{1}$ Optics Laboratory, School of Engineering, École Polytechnique Fédérale de Lausanne (EPFL), \\ BM 4.107, Station 17, CH-1015 Lausanne, Switzerland \\ ${ }^{2}$ Department of Electrical Engineering, California Institute of Technology, 1 \\ 200 East California Boulevard, MC 136-93, Pasadena, California 91125, USA
}

(Received 7 January 2010; published 19 May 2010)

\begin{abstract}
A nonlinear optical plasmonic core-shell nanocavity is demonstrated as an efficient, subwavelength coherent light source through second-harmonic generation. The nonlinear optical plasmonic nanocavity incorporates a noncentrosymmetric medium, which utilizes the entire mode volume for even-order nonlinear optical processes. In previous plasmonic nanocavities, enhancement of such processes was only possible at the interface but symmetry prohibited in the body. We measured an enhancement of over 500 times in the second-harmonic radiation power. Calculations show that an enhancement of over 3500 times is achievable.
\end{abstract}

DOI: 10.1103/PhysRevLett.104.207402

Nonlinear optical (NLO) harmonic generation [1] is important in a broad range of technologies and has become of increasing interest in photonics [2-4], chemistry [5,6], material science [7], and biosensing [8,9]. The interest was recently renewed [10-17] by the increasing demands for subwavelength coherent light sources, a critical component in the sciences of physics, information, and biology involving nanometer length scale. As one of the most important means for this purpose, second-harmonic generation (SHG) is prohibited in bulk centrosymmetric materials, though nanoscale SHG was often achieved by breaking the symmetry through interfaces [18], imperfect spheres [19], and asymmetric shapes [20]. Nanoscale SHG is also achieved though the used of noncentrosymmetric nanocrystals [11,13-16,21], asymmetric geometric arrangements [22], and coating of NLO material over nanospheres [23]. Practically, however, NLO harmonic generation is generally inefficient at such a small scale. Plasmonic nanocavities are thus intriguing for the construction of more efficient coherent NLO light sources because of their compact mode volume [24] resulting in a highly concentrated local field at resonance [25]. We have synthesized plasmonic core-shell nanocavities using a noncentrosymmetric NLO cavity medium, which enables the entire mode volume of the cavity to harness the enhanced local field. This results in an intrinsically NLO plasmonic nanocavity that is fundamentally different from previous dielectric core plasmonic nanoshells [26], where intracavity SHG is symmetry prohibited, and the plasmonic enhancement in NLO harmonic generation is so far limited to the interface $[23,27]$. Our calculations show that a geometry-tunable enhancement factor of up to several thousands in the SHG efficiency is theoretically achievable in these NLO plasmonic nanocavities. We experimentally demonstrate enhancements of 500 and 70 times under resonant and off-resonance conditions, respec-
PACS numbers: 78.67.Bf, 42.65.Ky, 73.20.Mf, 81.16.Dn

tively, using $100 \mathrm{~nm} \mathrm{BaTiO} / 3$ Au core-shell structures. The results agree well with the theoretical prediction.

The principle of intracavity SHG enhancement in a plasmonic nanocavity is illustrated in Fig. 1(a). The structure consists of a noncentrosymmetric crystalline core (tetragonal $\mathrm{BaTiO}_{3}$ as an example) of radius $r_{1}$ enclosed in a plasmonic shell of thickness $t_{s}$. The core medium possesses a dielectric constant $\epsilon_{1}$ and a second-order nonlinear optical coefficient tensor $\mathbf{d}$. The metal shell has a frequency-dependent dielectric constant $\epsilon_{2}$, which, in a surrounding medium of dielectric constant $\epsilon_{3}$, gives rise to a resonance at frequency $\omega$. Under the excitation of an external optical field $\mathbf{E}_{0}$ at the resonant frequency, the internal field and the induced material polarization $\mathbf{p}$ are significantly enhanced, which results in an enhancement in the internal second-harmonic emission. If the enhancement of the internal emission is greater than the attenuation of the shell, a net gain in SHG is obtained. A particularly important feature of the resonantly enhanced SHG is its tunability inherited from the plasmonic core-shell nanostructure. Figure 1(b) shows example calculations of the SHG radiation power as a function of the excitation frequency for three core-shell nanostructures.

The SHG enhancement inside the cavity can be found by comparing the electric field inside the SHG nanocrystal with the metal shell, $E_{1}^{(s)}$, to that without the shell, $E_{1}^{(b)}$. The factor of enhancement on the total SHG emission power is then $\Gamma_{\mathrm{SHG}}^{(i)}=\left[E_{1}^{(s)} / E_{1}^{(b)}\right]^{4}$. This 4 th-order dependence gives rise to a large enhancement in the SHG emission even with a moderate enhancement of the electric field in the core region, which is a much stronger effect than the "twophoton resonance" $[18,28,29]$. The internal field is often evaluated through the electrostatic approximation [25], which elegantly explains the resonance behavior of the core-shell nanostructures. However, we found it often nec- 


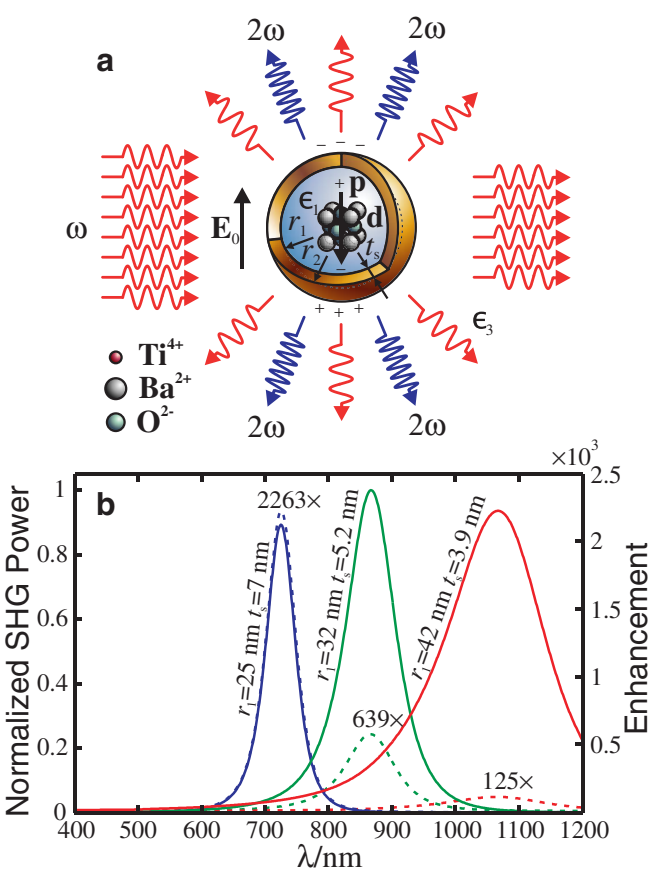

FIG. 1 (color online). Enhancement and tunability of plasmonic SHG nanocavities. (a) Principle and geometry of plasmonic SHG nanocavities. (b) Example calculation with various structural parameters demonstrating the tunability of plasmonic SHG nanocavities. Solid curves represent radiation power in second-harmonic frequency normalized to the maximum radiation power among the three examples. Dotted curves represent the factor of SHG enhancement compared to the core.

essary to employ the full Mie theory of electromagnetic scattering of core-shell structures [30] for the accurate calculation of the resonance frequency and the enhancement of the internal field. Our Mie-theory calculations [31] show that the electrostatic approximation overestimates the internal field enhancement when the shell is thick or the core size is large.

The transmission $T_{s}$ of the SHG emission through the $\mathrm{Au}$ shell, which is nonresonant, was modeled as a plane wave propagating through a planar gold film of the same thickness and verified by finite difference time domain simulations in a two-dimensional geometry [31]. The overall enhancement of the SHG emission is then $\Gamma_{\mathrm{SHG}}=$ $\Gamma_{\mathrm{SHG}}^{(i)} T_{s}$. In order to emphasize the role of the shell thickness, we plot $\Gamma_{\mathrm{SHG}}$ in Fig. 2 as a function of $t_{s}$, while the corresponding core size $r_{1}$ and the ratio $r_{1} / r_{2}$ can be found in [31]. At the thin shell limit, the surface electron scattering in the gold shell results in a low cavity $Q$ factor. At the thick shell limit, the shielding effect of the gold shell dominates. This results in an optimum thickness at which maximum enhancement is achieved. This optimum point is weakly dependent on the resonance frequency and ranges from 6.5 to $8.5 \mathrm{~nm}$ within the frequency range calculated here. The largest enhancement in the calculations is about $3.5 \times 10^{3}$ at the resonance wavelength of $700 \mathrm{~nm}$, although

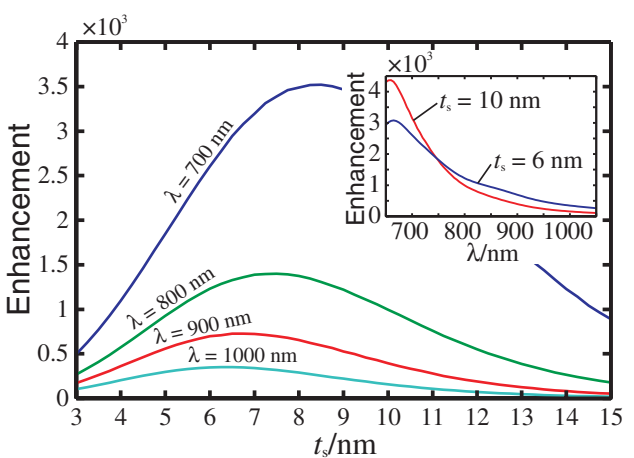

FIG. 2 (color online). Factor of SHG emission enhancement as a function of the shell thickness for different resonance frequencies (marked as wavelength in vacuum). The calculations are based on $\mathrm{BaTiO}_{3} / \mathrm{Au}$ core-shell structures. The inset shows the factor of enhancement as a function of resonance frequency when the shell thickness is tuned to $6 \mathrm{~nm}$ (blue curve) and $10 \mathrm{~nm}$ (green curve).

it is not the theoretical limit. Our calculations also reveal that $\Gamma_{\mathrm{SHG}}$ decreases with increasing resonance wavelength (which requires a larger core), as shown in the inset of Fig. 2. When the effective electromagnetic mode volume and the cavity $Q$ factor are taken into account, $\Gamma_{\mathrm{SHG}}$ is analogous with the Purcell factor [32] for spontaneous emission.

We adapted previous self-assembly schemes for silica core-gold-shell nanostructure [27] to the synthesis of $\mathrm{BaTiO}_{3} / \mathrm{Au}$ nanocavities. The principle and the experimental detail of the synthesis of such nanostructures are described in [31]. Figure 3 plots the measured ensemble extinction spectrum of the $\mathrm{BaTiO}_{3} / \mathrm{Au}$ nanocavities. The plasmonic resonance peak was roughly tuned to $800 \mathrm{~nm}$ wavelength to work with Ti:sapphire oscillators. Because of the large size variation of the core [31], the resonance shows a wide inhomogeneous broadening.

The SHG property of nanocrystals was quantified in terms of a "second-harmonic scattering cross section," which is defined as $W_{p}^{(2 \omega)}=\sigma^{(2 \omega)} I_{0}^{2}$, where $W_{p}^{(2 \omega)}$ is the total radiation power in the doubled frequency, $\sigma^{(2 \omega)}$ is the

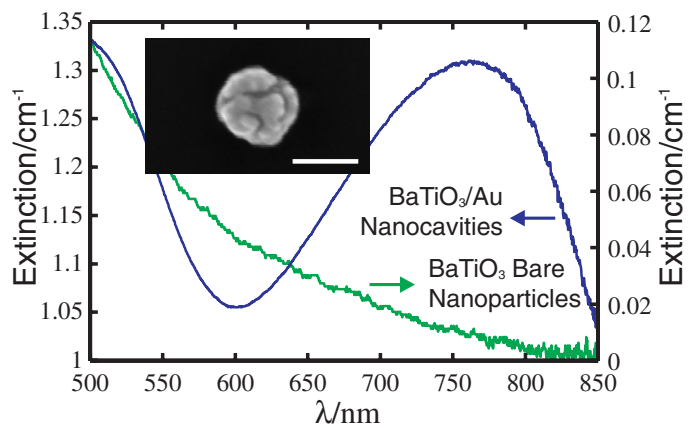

FIG. 3 (color online). Measured ensemble extinction spectrum of $\mathrm{BaTiO}_{3} / \mathrm{Au}$ core-shell nanocavities (blue curve) and the bare $\mathrm{BaTiO}_{3}$ nanocrystals (green curve). The inset shows a typical final product of the core-shell growth. The scale bar is $100 \mathrm{~nm}$. 

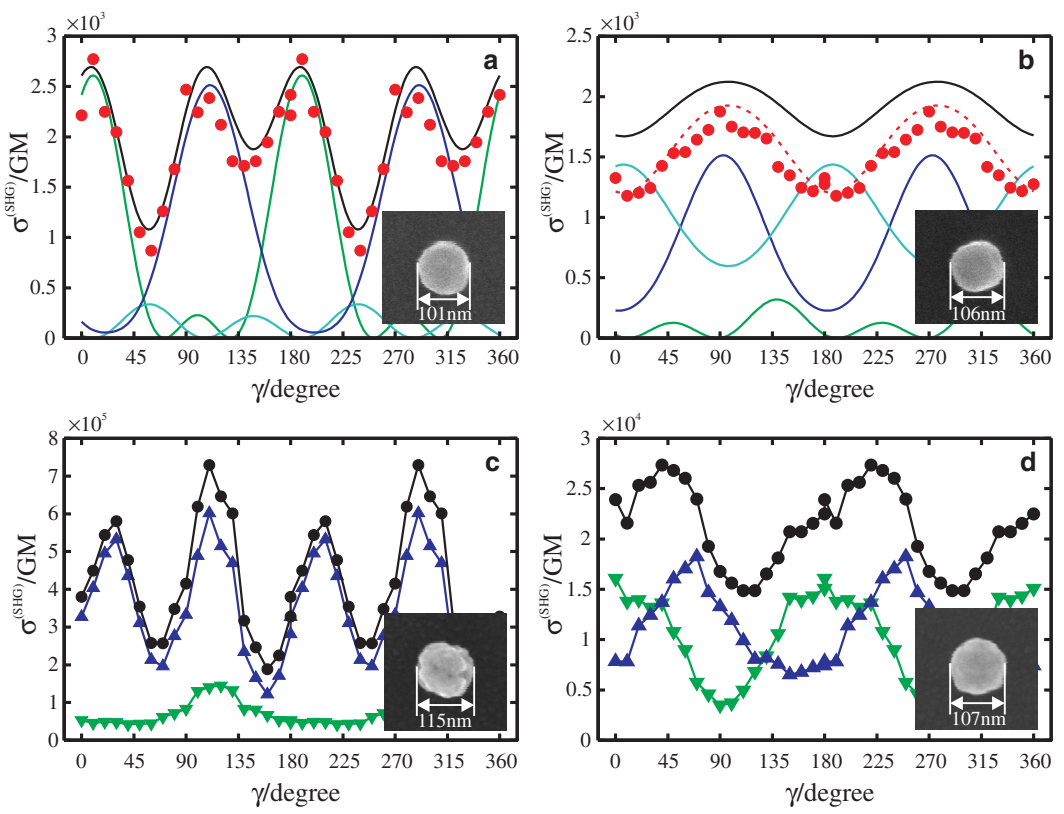

FIG. 4 (color online). Measurement of the SHG enhancement in the plasmonic SHG nanocavities. (a) and (b) Calculated and measured SHG scattering cross sections of the bare $\mathrm{BaTiO}_{3}$ nanocrystal shown in the insets. Red dots: measurement data. Blue, green, and cyan curves: calculated $x, y$, and $z$ components of the cross section. Black curve: total cross section. The $20 \%$ mismatch in (b) was due to the inability to distinguish the axial component from the transverse component experimentally. The red dotted curve shows calculations treating axial and transverse components equally. (c) and (d) Measured SHG scattering cross section of the $\mathrm{BaTiO}_{3} / \mathrm{Au}$ core-shell nanocavity shown in the insets. Blue up triangles and green down triangles: measurements in $x$ and $y$ polarization, respectively. Black circles: total cross section. second-harmonic scattering cross section, and $I_{0}$ is the excitation intensity. Following the convention of two-photon absorption [33], we use Göppert-Mayer (GM) as the unit for $\sigma^{(2 \omega)}$, where $1 \mathrm{GM}=1 \times 10^{-50} \mathrm{~cm}^{4} \mathrm{~s}_{\text {photons }}{ }^{-1}$.

Before a quantified $\Gamma_{\text {SHG }}$ can be measured, we must establish a model for the SHG emission power from a single bare SHG nanosphere. Following our previous approach [14], the internal electric field can be expressed as [34] $\mathbf{E}_{1}=\left[3 \epsilon_{3} /\left(\epsilon_{1}+2 \epsilon_{3}\right)\right] \mathbf{E}_{0}$, where material birefringence is ignored. The nonlinear polarization at the doubled frequency is then $\mathbf{p}^{(2 \omega)}=(4 / 3) \pi r^{3}\left[3 \epsilon_{3} /\left(\epsilon_{1}+2 \epsilon_{3}\right)\right]^{2} \mathbf{d}$. $\mathbf{E}_{0} \mathbf{E}_{0}$, which is treated as an electric dipole. Because of the tensor nature of the SHG process, this dipole is arbitrarily oriented in the laboratory reference frame. The total radiation power produced by the dipole over the entire $4 \pi$ solid angle is [34] $\mathbf{W}_{p}^{(2 \omega)}=\left(c^{2} Z_{0} k_{2}^{4} / 12 \pi\right)\left|\mathbf{p}^{(2 \omega)}\right|^{2}$, where $c$ is the speed of light, $Z_{0}$ is the impedance of vacuum, and $k_{2}=2 \omega / c$. We use a vector to mark the radiation power in order to separate the radiations from the $x, y$, and $z$ component of the dipole. The second-harmonic scattering cross section is then

$$
\boldsymbol{\sigma}^{(2 \omega)}=\frac{16 \pi}{27} \frac{1}{\epsilon_{1}}\left(\frac{3 \epsilon_{1}}{\epsilon_{3}+2 \epsilon_{1}}\right)^{4} c^{2} Z_{0}^{3} k_{2}^{4} r^{6}\left|\mathbf{d} \cdot \hat{\mathbf{e}}_{0} \hat{\mathbf{e}}_{0}\right|^{2},
$$

where $\hat{\mathbf{e}}_{0}$ is the unit vector of $\mathbf{E}_{0}$ in the crystal reference frame. The overall cross section is the sum of the three components. Here we use $I_{0}=n_{3}\left|\mathbf{E}_{0}\right|^{2} /\left(2 Z_{0}\right)$, where $n_{3}=\epsilon_{3}^{1 / 2}$.

Equation (1) was verified through correlated scanning electron microscopy (SEM) and optical measurements using a femtosecond Ti:sapphire oscillator operated at $800 \mathrm{~nm}$ for excitation. Figures 4(a) and 4(b) show the measured SHG cross section (red dots) as a function of $\gamma$, the polarization angle of the excitation field. The crystal orientation (not shown) was determined by calculating the SHG response using Eq. (1) for all orientations and finding the best match to the measurement data. A discussion on the measurement of an arbitrarily oriented dipole using high numerical aperture objective can be found in [31]. The measurements show that our model agrees well with the experimental data. We thus established a reference standard using the SHG cross section of an arbitrarily oriented $100 \mathrm{~nm} \mathrm{BaTiO}_{3}$, which is $1.4 \times 10^{3} \mathrm{GM}$ minimum and $2.0 \times 10^{3} \mathrm{GM}$ maximum.

To quantify the enhancement of $\mathrm{SHG}$, we performed correlated SEM-SHG measurements of isolated $\mathrm{BaTiO}_{3} / \mathrm{Au}$ nanocavities [31] under resonant and offresonance conditions, as shown in Figs. 4(c) and 4(d), respectively. At the excitation wavelength of $800 \mathrm{~nm}$, the maximum SHG cross section in Fig. 4(c) is $7.3 \times 10^{5} \mathrm{GM}$. Assuming a shell thickness of $10 \mathrm{~nm}$, as roughly suggested by the SEM images, the core diameter of the measured nanocavity is estimated to be $95 \mathrm{~nm}$. Projecting the measurement to the $100 \mathrm{~nm}$ standard core size, we estimate the cross section to be $1.0 \times 10^{6} \mathrm{GM}$, which is 500 and 710 times the previously calculated maximum and minimum value for the bare $100 \mathrm{~nm} \mathrm{BaTiO}_{3}$ nanocrystal, respectively. Given the constraint of the outer diameter of $115 \mathrm{~nm}$, this value was found in good agreement with the theoretical prediction of 540 times for a structure of $r_{1}=$ $46 \mathrm{~nm}$ and $r_{2}=57.5 \mathrm{~nm}$, which resonates at $800 \mathrm{~nm}$ wavelength. The maximum cross section in Fig. 4(d) is $2.7 \times 10^{3} \mathrm{GM}$, and the enhancement ranges from 52 to 74 times. Given the outer diameter of $107 \mathrm{~nm}$, this was again in good agreement with the theoretical prediction of 110 times for $r_{1}=41 \mathrm{~nm}$ and $r_{2}=53.5 \mathrm{~nm}$. Further calculations show that its resonance peak is located at $733 \mathrm{~nm}$ wavelength where an enhancement factor of 703 times is achievable. 


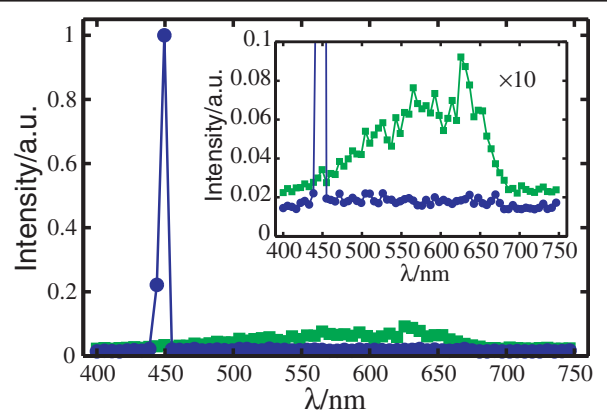

FIG. 5 (color online). Emission spectra of nominally $100 \mathrm{~nm}$ $\mathrm{BaTiO}_{3} / \mathrm{Au}$ core-shell nanocavities (blue solid circles) and $200 \mathrm{~nm}$ gold nanoparticles (green solid squares) normalized to the SHG peak intensity of the $\mathrm{BaTiO}_{3} / \mathrm{Au}$ core-shell structures. The blue and green solid lines are for visual aid. The inset shows the same data magnified by 10 in magnitude, where the twophoton photoluminescence of $200 \mathrm{~nm}$ gold nanoparticles is more visible.

To verify whether the strong SHG emission originated from the surface of gold rather than from the NLO core, we performed spectral measurements for both the 100-nm SHG nanocavities and 200-nm solid gold nanoparticles under identical conditions [31]. We used larger solid nanoparticles since $100 \mathrm{~nm}$ solid gold nanoparticles were unable to generate sufficient signal under the condition required for the SHG measurement. The excitation wavelength was tuned to $900 \mathrm{~nm}$. As seen in Fig. 5, a strong SHG signal was detected in the nanocavities, while only two-photon photoluminescence was detected in the solid gold nanoparticles. Scaling rules based on Mie calculations and previous hyper-Rayleigh scattering results suggest that the surface contribution to the SHG response in the NLO plasmonic nanocavities does not exceed 5\% [31] and is thus negligible.

The striking optical property of the NLO plasmonic nanocavities will enable devices and applications that are heretofore beyond reach (comparisons between NLO plasmonics nanocavities and other NLO materials can be found in [31]). As nanoprobes, the NLO nanocavities will open up new avenues in biomedical imaging applications $[14,35]$. They can also be assembled into a regular matrix to construct nonlinear photonic crystal $[2,3]$ or nonlinear metamaterials. By encapsulating proper nonlinear cavity media, this work provides a new platform for nanoscale coherent photon sources through a variety of NLO processes, including second- and third-harmonic generation, sum and difference frequency generation, and coherent anti-Stokes Raman scattering. Furthermore, the large polarizability of the plasmonic shell combined with the high NLO susceptibility will likely introduce new nonlinear modulation pathways for photon-photon and electron-photon interactions.

This work was supported by Swiss National Center of Competence in Research (NCCR), Quantum Photonics.
We thank Paul Bowen of the Powder Technology Laboratory at EPFL for kindly supplying $\mathrm{BaTiO}_{3}$ nanocrystals to us.

*ye.pu@epfl.ch

[1] P. A. Franken et al., Phys. Rev. Lett. 7, 118 (1961).

[2] V. Berger, Phys. Rev. Lett. 81, 4136 (1998).

[3] N. G. R. Broderick et al., Phys. Rev. Lett. 84, 4345 (2000).

[4] B. Corcoran et al., Nat. Photon. 3, 206 (2009).

[5] R. M. Corn and D. A. Higgins, Chem. Rev. 94, 107 (1994).

[6] A. Zumbusch, G. R. Holtom, and X. S. Xie, Phys. Rev. Lett. 82, 4142 (1999).

[7] S. I. Stupp et al., Science 276, 384 (1997).

[8] P. J. Campagnola and L. M. Loew, Nat. Biotechnol. 21, 1356 (2003).

[9] C.L. Evans et al., Proc. Natl. Acad. Sci. U.S.A. 102, 16807 (2005).

[10] Y. Nakayama et al., Nature (London) 447, 1098 (2007).

[11] M. Zielinski et al., Small, 5, 2835 (2009).

[12] F. W. Vance, B. I. Lemon, and J. T. Hupp, J. Phys. Chem. B 102, 10091 (1998).

[13] L. Bonacina et al., Appl. Phys. B 87, 399 (2007).

[14] C. L. Hsieh et al., Opt. Express 17, 2880 (2009).

[15] L. L. Xuan et al., Appl. Phys. Lett. 89, 121118 (2006).

[16] A. B. Djurisic and Y. H. Leung, Small 2, 944 (2006).

[17] M. Danckwerts and L. Novotny, Phys. Rev. Lett. 98, 026104 (2007).

[18] J. I. Dadap et al., Phys. Rev. Lett. 83, 4045 (1999).

[19] J. Nappa et al., Phys. Rev. B 71, 165407 (2005).

[20] A. K. Singh et al., Chem. Phys. Lett. 481, 94 (2009).

[21] M. Jacobsohn and U. Banin, J. Phys. Chem. B 104, 1 (2000).

[22] B. K. Canfield et al., Nano Lett. 7, 1251 (2007).

[23] X. Vidal et al., Opt. Lett. 33, 699 (2008).

[24] S. A. Maier, Opt. Express 14, 1957 (2006).

[25] A. E. Neeves and M.H. Birnboim, J. Opt. Soc. Am. B 6, 787 (1989).

[26] S. J. Oldenburg et al., Chem. Phys. Lett. 288, 243 (1998).

[27] S. J. Oldenburg et al., J. Chem. Phys. 111, 4729 (1999).

[28] G. S. Agarwal and S. S. Jha, Solid State Commun. 41, 499 (1982).

[29] R. C. Johnson et al., Chem. Phys. Lett. 356, 534 (2002).

[30] C. F. Bohren and D. R. Huffman, Absorption and Scattering of Light by Small Particles (Wiley, New York, 1998).

[31] See supplementary material at http://link.aps.org/ supplemental/10.1103/PhysRevLett.104.207402 for information about synthesis of the $\mathrm{BaTiO}_{3}$-Au nanocavities, the theoretical calculation of the internal field, and experimental details.

[32] E. M. Purcell, Phys. Rev. 69, 681 (1946).

[33] C. Xu et al., Proc. Natl. Acad. Sci. U.S.A. 93, 10763 (1996).

[34] J.D. Jackson, Classical Electrodynamics (Wiley, New York, 1998).

[35] A. V. Kachynski et al., J. Phys. Chem. C 112, 10721 (2008). 